\title{
Thiamine and Pyridoxine Alleviate Oxidative Damage by Copper Stress in Green Alga Chlorella vulgaris
}

\author{
A. A. Farghl \\ Department of Botany, Faculty of Science at Qena, South \\ Valley University, Qena, Egypt.
}

\begin{abstract}
7 HIAMINE and pyridoxine were investigated for their capacity to alleviate oxidative damage by copper stress on a local alga $(C$. vulgaris). Lower levels of $\mathrm{Cu}^{+2}$ induced a slight stimulation in growth criteria (growth rate \& dry weight), photosynthetic pigments $(0.5 \mu \mathrm{M})$ and $\mathrm{O}_{2}$-evolution $(0.5,10 \mu \mathrm{M})$ of $C$. vulgaris which were inhibited by high $\mathrm{Cu}^{+2}$ concentrations. In contrast, $\mathrm{O}_{2}$-uptake was retarded at the lower $\mathrm{Cu}^{+2}$ levels then significantly increased by increasing the $\mathrm{Cu}^{+2}$ in algal cultures. Proline, MDA contents, and antioxidant enzyme activity of $C$. vulgaris markedly increased under $\mathrm{Cu}^{+2}$ stress. On the other hand, addition of thiamine or pyridoxine alleviated the oxidative damage of $\mathrm{Cu}^{+2}$ on $C$. vulgaris growth and enhanced growth, pigment contents, $\mathrm{O}_{2}$ - evolution, and antioxidant enzyme activity in the algal cultures compared to reference controls. While $\mathrm{O}_{2}$-uptake, proline content, and lipid peroxidation levels were decreased, thiamine or pyridoxine scavenger systems might be important for supporting the ability of $C$. vulgaris to resist copper toxicity.
\end{abstract}

Keywords: Copper sulfate, Green alga, Thiamine, Pyridoxine, Antioxidant enzyme, Lipid peroxidation, Proline.

Microalgae are sensitive indicators of environmental change and, as the basis of most freshwater and marine ecosystems, are widely used in the assessment of risk and development of environmental regulations for metals (Levy et al., 2007). $\mathrm{Cu}^{+2}$ is essential for macroalgae, and at low concentrations participates in important biological reactions as an enzymatic cofactor and electron carrier in photosynthetic and respiratory processes. For example, $\mathrm{Cu}^{+2}$ is required as a cofactor of superoxide dismutase (EC 1.1.5.1.1) (Andrade et al., 2004). Copper can interfere with numerous physiological processes and is considered to be potentially cytotoxic when applied in higher amounts, and its toxicity varies among different macroalgae (Chang \& Sibley, 1993). The toxicity of copper is mainly related to free ions and is it a potent inhibitor of photosynthesis in microalgae (Küpper et al., 2002). Excess $\mathrm{Cu}^{+2}$ in plant cells may activate molecular oxygen and generate reactive oxygen species (ROS) (Wang et al., 2004 and Li et al., 2010). Cu-induced generation of hydrogen peroxide, hydroxyl radicals, and other ROS has been directly correlated with damage to membrane lipids and proteins (Gupta \& Kalra, 2006). This toxic effect coming from the cellular oxidative state may be allayed by several antioxidative systems such as superoxide dismutase (SOD), catalase and peroxidase (POD) (Joseph \& Jini, 2010).

E-mail: farghla@yahoo.com 
Peroxidases may remove excess $\mathrm{H}_{2} \mathrm{O}_{2}$ caused by metal stress. They are involved in several physiological and biochemical processes, such as cell growth and expansion (Fang \& Kao, 2000), auxin catabolism (Passsardi et al., 2004), and lignification (Brownleader et al., 2000).

Proline accumulates in several plants under stress, providing the plants with protection against damage by ROS. Proline plays important roles in osmoregulation (Laliberte \& Hellebust, 1989), protection of enzymes (Nikolopoulos \& Manetas, 1991), stabilization of the machinery of protein synthesis (Kadpal \& Rao, 1985), regulation of cytosolic acidity (Venekemp, 1989), and scavenging of free radicals (Smirnoff \& Cumbes, 1989). It also acts as an effective singlet oxygen quencher (Alia et al., 2001). Malondialdehyde (MDA) is a cytotoxic product of lipid peroxidation and an indicator of free radical production and consequent tissue damage (Ohkawa et al., 1979).

Vitamins are organic compounds that are required in trace amounts to maintain normal growth and proper development of organisms. These compounds act as coenzymes and thus take essential part in the regulation of metabolism. Vitamins, can be limiting factors in plant development (Hassanein et al., 2009). Thiamine (vitamin B1) is a necessary ingredient for the biosynthesis of the coenzyme Thiamine pyrophosphate, so it plays an important role in carbohydrate metabolism. It is an essential nutrient for both plants and animals (Kawasaki, 1992). Pyridoxine (vitamin B6) is a water-soluble vitamin and is part of the vitamin B complex group. Several forms of the vitamin are known, but pyridoxal phosphate (PLP) is the active form and is a cofactor in many reactions of amino acid metabolism, including transamination, deamination, and decarboxylation. PLP also is necessary for the enzymatic reaction governing the release of glucose from glycogen.

\section{Materials and Methods}

\section{Organism and culture conditions}

Axenic cultures of Chlorella vulgaris (a unicellular and non-motile green microalga) were isolated from soil of Qena Governorate, Egypt. All experiments were carried out in $500 \mathrm{ml}$ Erlenmeyer flasks containing $100 \mathrm{ml}$ Bold's basal medium (Bischoff \& Bold, 1963), and incubated at temperature $25 \pm 1 \mathrm{C}^{\circ}, 30 \mu \mathrm{E} /$ $\mathrm{m}^{2} / \mathrm{s}$ (cool white fluorescent light), with $16 \mathrm{hr}$ light $/ 8 \mathrm{hr}$ dark period. The cultures were supplied with sterilized dry air and $\mathrm{CO}_{2}(97 \%: 3 \%$, v/v) for 7 days.

\section{Treatments}

Chlorella vulgaris Beijer cultured was amended to $200 \mathrm{mg} \mathrm{L}^{-1}$ of both thiamine and pyridoxine individually in the absence or presence of various levels of $\mathrm{Cu}^{+2}: 0.5,10,50,100$ and $200 \mu \mathrm{M}$ in the form of copper sulfate. The control (o) was absolutely $\mathrm{Cu}^{+2}$ and vitamin free (only media).

\section{Measurements}

Dry weight of cells was taken after filtering and drying overnight at $105^{\circ} \mathrm{C}$.

Egypt. J. Microbiol. 47 (2012) 
Determination of the optical density of the green algal suspension was at $560 \mathrm{~nm}$ (Wetherel, 1961). The photosynthetic pigments (Chl.a,Chl.b and carotenoids) were determined using the spectrophotometric method recommended by Metzner et al. (1965). Oxygen evolution was determined using an oxygen meter (G867 with $\mathrm{O}_{2}$ electrode). Dark respiration (dark oxygen uptake) was determined as oxygen uptake in the dark, the system mentioned above for the estimating of oxygen evolution was used for estimating dark respiration. At the end of oxygen evolution measurements, all the lights were switched off and the flasks were wrapped tightly in aluminum foil for complete darkness. Proline content was determined according to Bates et al. (1973).

\section{Determination of antioxidant enzymes activity}

Algae samples were prepared as described in Mukherjee \& Choudhuri (1983). A fresh sample (500 mg) was frozen in liquid nitrogen and finely ground with a chilled pestle and mortar, the frozen powder was added to $10 \mathrm{ml} 100 \mathrm{mM}$ phosphate buffer $\left(\mathrm{KH}_{2} \mathrm{PO} 4 / \mathrm{K}_{2} \mathrm{HPO}_{4}, \mathrm{pH} 7.0\right)$ containing $0.1 \mathrm{mM} \mathrm{Na}_{2}$ EDTA and $0.1 \mathrm{~g}$ polyvinylpyrrolidone (PVP), the homogenate was filtered through cheese cloth then centrifuged at $15,000 \mathrm{~g}$ for $10 \mathrm{~min}$, the supernatant was recentrifuged at $18,000 \mathrm{~g}$ for $10 \mathrm{~min}$, and the resulted supernatant collected and stored at $4^{\circ} \mathrm{C}$ for assay of superoxide dismutase (SOD), catalase (CAT), peroxidase (POD) and ascorbate peroxidase (APX). $0.5 \mathrm{ml}$ of crude extract used for each enzyme assay.

SOD (EC 1.15.1.1) activity was measured according to Dhindsa \& Matowe (1981). CAT (EC 1.11.1.6) activity was measured as described in Aebi (1984) as the decrease of absorbance at $240 \mathrm{~nm}$ as a consequence of $\mathrm{H}_{2} \mathrm{O}_{2}$ consumption, and expressed according to Havir \& Mellate (1987). POD (EC 1.11.1.7) activity was determined according to Maehly \& Chance (1954). APX (EC 1.11.1.11) activity was determined as the decrease in absorbance of ascorbate at $290 \mathrm{~nm}$ as oxidised ascorbic acid (Asada \& Chen, 1992).

The level of lipid peroxidation was measured in terms of malondialdehyde (MDA) content using the method of Heath \& Packer (1968) as follows: to $2.0 \mathrm{ml}$ aliquot of the supernatant $4.0 \mathrm{ml}$ of $0.5 \%$ thiobarbituric acid (TBA) in $20 \%$ Trichloroacetic acid (TCA) was added. The mixture was heated at $95^{\circ} \mathrm{C}$ for $30 \mathrm{~min}$ and quickly cooled in an ice bath then centrifuged at $10000 \mathrm{~g}$ for $10 \mathrm{~min}$. The absorbance of supernatant was measured at $532 \mathrm{~nm}$. The value for nonspecific absorption at $600 \mathrm{~nm}$ was subtracted. The MDA content was calculated using its absorption coefficient of $155 \mathrm{nmol} \mathrm{cm}^{-1}$ and expressed as nmol (MDA) $\mathrm{g}^{-1}$ fresh matter.

\section{Statistical analysis}

The data were subjected to analysis by the least significant differences test (L.S.D) using PC-STATE program version 1A, coded by Rao,M: Blane, K and Zannenberg, M, University of Georgia. In each case the data were obtained from triplicate cultures and represented as means $\pm \mathrm{SD}$. 


\section{Results}

Effects of thiamine and pyridoxine on growth rate and dry weight

Table 1 shows that, there are variable results according to the concentration of the $\mathrm{Cu}^{+2}$ ions. There were no significant results in the growth rate (as optical density) and dry weight of $C$. vulgaris cultures at $0.5 \mu \mathrm{M} \mathrm{Cu}^{+2}$. Thereafter, increasing the $\mathrm{Cu}^{+2}$ concentration in the algal cultures medium reduced growth rate and dry weight about $73 \%$ and $71 \%$, respectively, compared to the control, when the level was $200 \mu \mathrm{M} \mathrm{Cu}^{+2}$.

Thiamine (vit.B1) or pyridoxine (vit.B6) treatments enhanced the growth and dry weight of $C$. vulgaris up to $10 \mu \mathrm{M} \mathrm{Cu}^{+2}$ and still alleviated the inhibitory effect of $\mathrm{Cu}^{+2}$ at $50 \mu \mathrm{M} \mathrm{Cu}^{+2}$, relative to the reference controls.

TABLE 1. Effects of thiamine and pyridoxine on growth rate and dry weight of $C$. vulgaris grown under copper $\left(\mathrm{Cu}^{+2}\right)$ stress.

\begin{tabular}{|c|c|c|c|c|c|}
\hline Treatments & $\begin{array}{l}\mathrm{Cu}^{+2} \\
(\mu \mathrm{M})\end{array}$ & $\begin{array}{l}\text { Optical } \\
\text { density }\end{array}$ & $\%$ & $\begin{array}{c}\text { dry weight } \\
\mu \mathrm{g} \mathrm{ml}^{-1}\end{array}$ & $\%$ \\
\hline \multirow{6}{*}{ Reference control } & 0 & $135+002$ & 10000 & $17067+067$ & 100 \\
\hline & 0.5 & $1.38 \pm 0.16$ & 102.22 & $175.79 \pm 0.17$ & 103.00 \\
\hline & 10 & $1.17^{* *} \pm 0.04$ & 86.66 & $145.00^{* * *} \pm 0.37$ & 84.96 \\
\hline & 50 & $0.96^{* *} \pm 0.06$ & 71.11 & $121.84^{* * *} \pm 0.97$ & 71.39 \\
\hline & 100 & $0.66^{* *} \pm 0.05$ & 48.88 & $95.97^{* * *} \pm 0.36$ & 56.23 \\
\hline & 200 & $0.37^{* \pm} \pm 0.06$ & 27.40 & $49.67^{* * *} \pm 1.42$ & 29.10 \\
\hline \multirow{6}{*}{$\begin{array}{l}\mathrm{Cu}^{+2}+200 \mathrm{mg} \mathrm{L}^{-1} \\
\text { thiamine }\end{array}$} & 0 & $1.78^{* * *} \pm 0.04$ & 131.85 & $210.85^{* 3 *} \pm 0.79$ & 123.54 \\
\hline & 0.5 & $1.60^{* *} \pm 0.09$ & 118.52 & $203.16^{* * *} \pm 0.42$ & 119.04 \\
\hline & 10 & $1.50^{*} \pm 0.06$ & 111.11 & $171.72 \pm 0.63$ & 100.62 \\
\hline & 50 & $1.24 \pm 0.12$ & 91.85 & $161.38 \pm 0.88$ & 94.56 \\
\hline & 100 & $0.98^{* *} \pm 0.11$ & 72.59 & $140.73^{* * *} \pm 0.37$ & 82.46 \\
\hline & 200 & $0.90^{* *} \pm 0.01$ & 66.66 & $119.20^{* * *} \pm 1.14$ & 69.84 \\
\hline \multirow{6}{*}{$\begin{array}{l}\mathrm{Cu}^{+2}+200 \mathrm{mg} \mathrm{L}^{-1} \\
\text { pyridoxine }\end{array}$} & 0 & $1.92^{* *} \pm 0.05$ & 142.22 & $221.00^{* * *} \pm 0.84$ & 129.49 \\
\hline & 0.5 & $1.66^{* *} \pm 0.06$ & 122.96 & $203.89^{* * *} \pm 0.37$ & 119.46 \\
\hline & 10 & $1.52^{* *} \pm 0.15$ & 112.59 & $173.44 \pm 0.62$ & 101.62 \\
\hline & 50 & $1.30 \pm 0.04$ & 96.30 & $162.72 \pm 0.36$ & 95.34 \\
\hline & 100 & $1.06^{* *} \pm 0.06$ & 78.52 & $142.77^{* * *} \pm 0.47$ & 83.65 \\
\hline & 200 & $0.92^{* *} \pm 0.08$ & 68.81 & $116.08^{* * *} \pm 0.72$ & 68.01 \\
\hline \multirow[t]{2}{*}{\begin{tabular}{|l|} 
L.S.D \\
\end{tabular}} & $5 \%$ & 0.12 & & 9.85 & \\
\hline & $1 \%$ & 0.16 & & 13.27 & \\
\hline
\end{tabular}

"Significant differences at $(\mathrm{p}=0.05)$ " highly significant differences from control at $(\mathrm{p}=0.01) . \pm \mathrm{SD}, \mathrm{n}=3$

Effects of thiamine and pyridoxine on photosynthetic pigments

Table 2 shows that, $0.5 \mu \mathrm{M} \mathrm{Cu}^{+2}$ slightly increased in the concentrations of Chl.a, Chl.b, carotenoids and the total pigments. Above $0.5 \mu \mathrm{M} \mathrm{Cu}^{+2}$ these values decreased significantly as the concentration of $\mathrm{Cu}^{+2}$ increased. The reduction in the Chl.a, Chl.b, carotenoids, and the total pigments were about 78\%, 78\%, $66 \%$ and $76 \%$, respectively compared to the absolute control.

Egypt. J. Microbiol. 47 (2012) 
TABLE 2. Effects of thiamine and pyridoxine on photosynthetic pigments (Chl.a, Chl.b and carotenoids) of $C$. vulgaris grown under copper $\left(\mathrm{Cu}^{+2}\right)$ stress.

\begin{tabular}{|c|c|c|c|c|c|c|c|c|c|}
\hline Treatment & $\begin{array}{l}\mathrm{Cu}^{+2} \\
(\mu \mathrm{M})\end{array}$ & $\begin{array}{l}\text { Chlo.a } \\
\mu \mathrm{g} \mathrm{ml}^{-1}\end{array}$ & $\%$ & $\begin{array}{l}\text { Chlo.b } \\
\mu \mathrm{g} \mathrm{ml}^{-1}\end{array}$ & $\%$ & $\begin{array}{c}\text { Car. } \\
\mu \mathrm{g} \mathrm{ml}^{-1}\end{array}$ & $\%$ & $\begin{array}{c}\text { Total } \\
\mu \mathrm{g} \mathrm{ml^{-1 }}\end{array}$ & $\%$ \\
\hline \multirow{6}{*}{$\begin{array}{l}\text { Reference } \\
\text { control }\end{array}$} & 0 & $6.04 \pm 0.06$ & 100.00 & $3.11 \pm 0.12$ & 100.00 & $1.91 \pm 0.11$ & 100.00 & $11.06 \pm 0.16$ & 100.00 \\
\hline & 0.5 & $6.35 \pm 0.05$ & 105.13 & $3.26 \pm 0.32$ & 104.82 & $2.1 \pm 0.17$ & 109.95 & $11.71 \pm 0.19$ & 106.88 \\
\hline & 10 & $5.21^{* *} \pm 0.17$ & 86.26 & $2.64^{* *} \pm 0.27$ & 84.89 & $1.75 \pm 0.32$ & 91.62 & $9.60^{* *} \pm 0.38$ & 86.80 \\
\hline & 50 & $4.37^{* *} \pm 0.17$ & 72.35 & $2.12^{* *} \pm 0.22$ & 68.17 & $1.50^{*} \pm 0.15$ & 78.53 & $7.99^{* *} \pm 0.17$ & 72.24 \\
\hline & 100 & $2.59^{* *} \pm 0.40$ & 42.88 & $1.25^{* *} \pm 0.12$ & 40.19 & $1.10^{* *} \pm 0.18$ & 57.59 & $4.94^{* *} \pm 1.54$ & 44.67 \\
\hline & 200 & $1.34^{* *} \pm 0.16$ & 22.19 & $0.68^{* *} \pm 0.14$ & 21.86 & $0.65^{* *} \pm 0.01$ & 34.03 & $2.67^{* *} \pm 0.15$ & 24.14 \\
\hline \multirow{6}{*}{$\begin{array}{l}\mathrm{Cu}^{+2}+200 \\
\mathrm{mg} \mathrm{L}^{-1} \\
\text { thiamine }\end{array}$} & 0 & $7.32^{* *} \pm 0.09$ & 121.19 & $4.21^{* *} \pm 0.18$ & 135.37 & $2.72^{* *} \pm 0.48$ & 142.41 & $14.25^{* *} \pm 0.58$ & 128.84 \\
\hline & 0.5 & $7.16^{* *} \pm 0.37$ & 118.54 & $3.81^{* *} \pm 0.28$ & 122.51 & $2.55^{* * *} \pm 0.57$ & 133.51 & $13.52^{* * *} \pm 0.95$ & 122.24 \\
\hline & 10 & $6.67^{*} \pm 0.18$ & 110.43 & $3.51^{*} \pm 0.10$ & 112.86 & $2.21 \pm 0.20$ & 115.71 & $12.39^{*} \pm 0.44$ & 112.02 \\
\hline & 50 & $5.38^{*} \pm 0.12$ & 89.07 & $2.70^{*} \pm 0.19$ & 86.82 & $1.63 \pm 0.17$ & 85.34 & $9.71^{*} \pm 0.23$ & 87.27 \\
\hline & 100 & $4.47^{* *} \pm 0.54$ & 74.03 & $2.13^{* *} \pm 0.20$ & 68.49 & $1.46 \pm 0.17$ & 76.44 & $8.06^{* *} \pm 0.29$ & 72.88 \\
\hline & 200 & $2.63^{* * *} \pm 0.10$ & 43.54 & $1.21^{* * *} \pm 0.10$ & 38.91 & $0.98^{* *} \pm 0.07$ & 51.31 & $4.82^{* *} \pm 0.07$ & 43.58 \\
\hline \multirow{6}{*}{$\begin{array}{l}\mathrm{Cu}^{+2}+200 \\
\mathrm{mg} \mathrm{L}^{-1} \\
\text { pyridoxine }\end{array}$} & 0 & $8.14^{* * *} \pm 0.13$ & 134.77 & $4.41^{* *} \pm 0.18$ & 141.80 & $2.66^{* *} \pm 0.38$ & 139.27 & $15.21^{* * *} \pm 0.53$ & 137.52 \\
\hline & 0.5 & $7.59^{* *} \pm 0.31$ & 125.66 & $4.23^{* *} \pm 0.15$ & 136.01 & $2.37^{*} \pm 0.93$ & 124.08 & $14.19^{* *} \pm 0.56$ & 128.30 \\
\hline & 10 & $7.24^{* *} \pm 0.34$ & 119.87 & $3.84^{*} \pm 0.28$ & 123.47 & $2.31 \pm 0.15$ & 120.94 & $13.39^{* *} \pm 0.70$ & 121.07 \\
\hline & 50 & $5.78 \pm 0.27$ & 95.69 & $2.91 \pm 0.09$ & 93.80 & $1.84 \pm 0.22$ & 96.34 & $10.53 \pm 0.38$ & 95.21 \\
\hline & 100 & $4.73^{* *} \pm 0.22$ & 78.31 & $2.32^{* *} \pm 0.29$ & 74.60 & $1.59 \pm 0.08$ & 83.25 & $8.64 * * \pm 0.09$ & 72.75 \\
\hline & 200 & $2.95^{* *} \pm 0.70$ & 48.84 & $1.42^{* *} \pm 0.10$ & 45.66 & $1.17^{* *} \pm 0.05$ & 61.26 & $5.54^{* *} \pm 0.73$ & 50.09 \\
\hline \multirow[t]{2}{*}{ L.S.D } & $5 \%$ & 0.57 & & 0.35 & & 0.46 & & 1.03 & \\
\hline & $1 \%$ & 0.76 & & 0.48 & & 0.62 & & 1.39 & \\
\hline
\end{tabular}

Significant differences at $(\mathrm{p}=0.05){ }^{* * *}$ highly significant differences from control at $(\mathrm{p}=0.01)$. $\pm \mathrm{SD}, \mathrm{n}=3$

Thiamine (vit.B1) enhanced pigment contents in the algal cultures by about $21 \%, 35 \%$ and $42 \%$ for Chl. a, Chl.b and carrotenoids, respectively compared to the reference controls. It could alleviate $\mathrm{Cu}^{+2}$ toxicity and enhanced total pigment contents in the algal cultures by $22 \%$ and $12 \%$ at 0.5 and $10 \mu \mathrm{M} \mathrm{Cu}{ }^{+2}$, respectively compared to the reference control.

Pyridoxine (vit.B6) treatments alleviated the inhibitory effect of $\mathrm{Cu}^{+2}$ and enhanced total pigment contents in the algal cultures by about 28 and $21 \%$ at 0.5 and $10 \mu \mathrm{M} \mathrm{Cu}^{+2}$, respectively, compared to the reference control.

Effects of thiamine and pyridoxine on $\mathrm{O}_{2^{-}}$evolution, $\mathrm{O}_{2^{-}}$uptake, proline and MDA contents

Data presented in Table 3 show the changes occurred photosynthetic rate (oxygen evolution), respiration (dark oxygen uptake), proline content and malondialdehyde (MDA) content.

Photosynthetic rate (oxygen evolution)

The copper treatment induced insignificant changes in the photosynthetic oxygen evolution up to $10 \mu \mathrm{M} \mathrm{Cu}{ }^{+2}$ and then a highly significant decrease $(>60 \%)$ by increasing the concentration of $\mathrm{Cu}^{+2}$ at $200 \mu \mathrm{M} \mathrm{Cu}^{+2}$. 
TABLE 3. Effects of thiamine and pyridoxine on $\mathrm{O}_{2}$ - evolution, $\mathrm{O}_{2}$ - uptake, proline and MDA of $C$. vulgaris grown under copper $\left(\mathrm{Cu}^{+2}\right)$ stress.

\begin{tabular}{|c|c|c|c|c|c|c|c|c|c|}
\hline Treatment & $\begin{array}{l}\mathrm{Cu}^{+2} \\
(\mu \mathrm{M})\end{array}$ & \begin{tabular}{|c|}
$\mathrm{O}_{2-}$ \\
evolution \\
mg L L $^{-1}$ \\
\end{tabular} & $\%$ & $\begin{array}{c}\mathrm{O}_{2} \text {-uptake } \\
\mathrm{mg} \mathrm{L}^{-1}\end{array}$ & $\%$ & $\begin{array}{l}\text { Proline } \\
\mu^{-1} \text { mg }^{-1} \\
\text { D.W. }\end{array}$ & $\%$ & $\begin{array}{c}\text { MDA } \\
\text { nmol g }^{-1} \\
\text { F.W. }\end{array}$ & $\%$ \\
\hline \multirow{6}{*}{$\begin{array}{l}\text { Reference } \\
\text { control }\end{array}$} & 0 & $5.17 \pm 0.55$ & 100.00 & $3.26 \pm 0.25$ & 100.0 & $0.53 \pm 0.07$ & 100.00 & $45.67 \pm 0.60$ & 100.00 \\
\hline & 0.5 & $5.84 \pm 0.61$ & 112.96 & $3.17 \pm 0.02$ & 97.24 & $0.55 \pm 0.01$ & 103.77 & $45.98 \pm 0.11$ & 100.68 \\
\hline & 10 & $5.22 \pm 0.05$ & 100.97 & $3.12 \pm 0.19$ & 95.71 & $0.60 \pm 0.01$ & 113.21 & $46.81 \pm 0.46$ & 102.50 \\
\hline & 50 & $4.01^{* *} \pm 0.10$ & 77.56 & $4.20^{* * *} \pm 0.15$ & 128.83 & $0.70^{* * *} \pm 0.01$ & 132.08 & $57.07^{* *} \pm 0.75$ & 124.96 \\
\hline & 100 & $3.26^{* *} \pm 0.06$ & 63.06 & $4.32^{* *} \pm 0.06$ & 132.52 & $0.85^{* *} \pm 0.04$ & 160.38 & $66.59^{* *} \pm 0.56$ & 145.81 \\
\hline & 200 & $1.62^{* * *} \pm 0.11$ & 31.33 & $4.61^{* *} \pm 0.12$ & 141.41 & $1.04^{* *} \pm 0.06$ & 196.23 & $78.12^{* *} \pm 0.84$ & 171.05 \\
\hline \multirow{6}{*}{$\begin{array}{l}\mathrm{Cu}^{+2}+200 \\
\mathrm{mg} \mathrm{L}^{-1} \\
\text { thiamine }\end{array}$} & 0 & $6.52^{* *} \pm 0.65$ & 126.11 & $3.25 \pm 0.02$ & 99.69 & $0.51 \pm 0.01$ & 96.23 & $48.01^{*} \pm 0.36$ & 105.12 \\
\hline & 0.5 & $6.11^{*} \pm 0.04$ & 118.08 & $3.15 \pm 0.16$ & 96.63 & $0.53 \pm 0.03$ & 100.00 & $40.93^{* *} \pm 0.72$ & 89.62 \\
\hline & 10 & $5.27 \pm 0.33$ & 101.93 & $2.97^{* *} \pm 0.14$ & 91.10 & $0.49 \pm 0.08$ & 92.45 & $44.32 \pm 0.98$ & 97.04 \\
\hline & 50 & $4.70 \pm 0.41$ & 90.91 & $3.21 \pm 0.24$ & 98.47 & $0.58 \pm 0.02$ & 109.43 & $47.53 \pm 0.80$ & 104.07 \\
\hline & 100 & $3.84^{* * *} \pm 0.22$ & 74.27 & $3.56^{* *} \pm 0.05$ & 109.20 & $0.67^{*} \pm 0.03$ & 126.42 & $54.52^{* *} \pm 1.09$ & 119.38 \\
\hline & 200 & $3.37^{* *} \pm 0.16$ & 65.18 & $3.67^{* *} \pm 0.11$ & \begin{tabular}{|l|}
112.58 \\
\end{tabular} & $0.74^{* *} \pm 0.02$ & 139.62 & $59.73^{* *} \pm 0.35$ & 130.79 \\
\hline \multirow{6}{*}{$\begin{array}{l}\mathrm{Cu}^{+2}+200 \\
\mathrm{mg} \mathrm{L}^{-1} \\
\text { pyridoxine }\end{array}$} & 0 & $6.76^{* *} \pm 0.66$ & 130.75 & $3.19 \pm 0.23$ & 97.87 & $0.44 \pm 0.01$ & 83.02 & $45.69 \pm 0.57$ & 100.04 \\
\hline & 0.5 & $6.18^{*} \pm 0.07$ & 119.54 & $3.01^{* * *} \pm 0.01$ & 92.33 & $0.42^{*} \pm 0.01$ & 79.25 & $38.48^{* *} \pm 0.58$ & 84.26 \\
\hline & 10 & $5.81 \pm 0.21$ & 112.38 & $3.03^{*} \pm 0.22$ & 92.94 & $0.47 \pm 0.01$ & 88.68 & $44.94 \pm 0.57$ & 98.40 \\
\hline & 50 & $4.71 \pm 0.40$ & 91.10 & $3.15 \pm 0.27$ & 96.63 & $0.53 \pm 0.01$ & 100.00 & $45.61 \pm 0.58$ & 99.87 \\
\hline & 100 & $4.01^{* * *} \pm 0.01$ & 77.56 & $3.00^{* *} \pm 0.06$ & 92.02 & $0.61 \pm 0.01$ & 115.09 & $55.17^{* *} \pm 0.85$ & 120.80 \\
\hline & 200 & $3.48^{* *} \pm 0.02$ & 67.31 & $3.60^{* *} \pm 0.04$ & 110.43 & $0.70^{* *} \pm 0.03$ & 132.08 & $60.95^{* *} \pm 0.63$ & 133.46 \\
\hline \multirow[b]{2}{*}{ L.S.D } & $5 \%$ & 0.76 & & 0.18 & & 0.11 & & 1.91 & \\
\hline & $1 \%$ & 1.02 & & 0.24 & & 0.15 & & 2.57 & \\
\hline
\end{tabular}

\section{Respiration (dark oxygen uptake)}

In contrast, at lower levels of copper stress, the dark $\mathrm{O}_{2}$-uptake gradual decreased, thereafter, it progressively increased to be about $41 \%$ over control at $200 \mu \mathrm{M} \mathrm{Cu}^{+2}$.

Application of both vitamins induce a marked stimulation in the $\mathrm{O}_{2-}$ evolution rate till $10 \mu \mathrm{M} \mathrm{Cu}{ }^{+2}$ with advantage to pyridoxine application compared to the corresponding stressed cultures. In contrast, $\mathrm{O}_{2}$-uptake inhibited at $0.5-10 \mu \mathrm{M} \mathrm{Cu}^{+2}$ levels, above there was gradual increase in $\mathrm{O}_{2}$-uptake in $C$. vulgaris cultures compared to the absolute control.

\section{Proline conten}

The proline content increased slightly up to $10 \mu \mathrm{M} \mathrm{Cu}{ }^{+2}$, then a sharply increased (about 97\%) as the concentration of $\mathrm{Cu}^{+2}$ increased at the level of $200 \mu \mathrm{M} \mathrm{Cu}^{+2}$ in relation to the control.

\section{Malondialdehyde (MDA) content}

On the other hand, a level of $10 \mu \mathrm{M} \mathrm{Cu}^{+2}$ induced insignificant changes in MDA content of $C$. vulgaris cultures, and then a highly significant accumulation. The highest increase was $71 \%$ over the control value at $200 \mu \mathrm{M} \mathrm{Cu}^{+2}$.

Egypt. J. Microbiol. 47 (2012) 
The supplementary two vitamins resulted in pronounced inhibition in the accumulation of proline and MDA contents compared to algae treated with only $\mathrm{Cu}^{+2}$, whatever the $\mathrm{Cu}^{+2}$ level used. Moreover the amount of proline and MDA remained mostly around those of control algae at the level of $50 \mu \mathrm{M} \mathrm{Cu}^{+2}$. The retarding effect was more pronounced in pyridoxine than in thiamine treated alga in the case of proline.

Effects of thiamine and pyridoxine on antioxidant enzymes activity (SOD, CAT, $P O D \& A P X)$ :

Activity of SOD, CAT and POD was markedly and progressively increased by increasing the concentration of $\mathrm{Cu}^{+2}$ in the algal cultures. SOD, CAT and POD activities reached maximum values about $179 \%, 200 \%$, and $173 \%$ of the absolute control, respectively in $C$. vulgaris treated with $200 \mu \mathrm{M} \mathrm{Cu}^{+2}$. On the other hand, the APX activity remained around the absolute control value at the all levels of $\mathrm{Cu}^{+2}$ (Table 4).

TABLE 4. Effects of thiamine and pyridoxine on antioxidant enzymes activity of $C$. vulgaris grown under copper $\left(\mathrm{Cu}^{+2}\right)$ stress.

\begin{tabular}{|c|c|c|c|c|c|c|c|c|c|}
\hline Treatments & $\begin{array}{c}\mathrm{Cu}^{+2} \\
(\mu \mathrm{M})\end{array}$ & \begin{tabular}{|c|} 
SOD \\
${\text { unit } \text { min }^{-1}}^{\mathbf{g}^{-1} \text { F.W. }}$ \\
\end{tabular} & $\%$ & $\begin{array}{c}\text { CAT } \\
{\text { unit } \text { min }^{-1}}^{\mathrm{g}^{-1} \text { F.W. }} \\
\end{array}$ & $\%$ & $\begin{array}{c}\text { POD } \\
{\text { unit } \text { min }^{-1}}^{\mathrm{g}^{-1} \text { F.W. }} \\
\end{array}$ & $\%$ & $\begin{array}{c}\text { APX } \\
\text { unit min }^{-1} \\
\text { g }^{-1} \text { F.W. }\end{array}$ & $\%$ \\
\hline \multirow{6}{*}{$\begin{array}{l}\text { Reference } \\
\text { control }\end{array}$} & 0 & $2.24 \pm 0.08$ & 100.00 & $3.07 \pm 0.05$ & 100.0 & $1.21 \pm 0.09$ & 100.00 & $0.82 \pm 0.01$ & 100 \\
\hline & 0.5 & $2.49^{*} \pm 0.12$ & 111.16 & $3.25 \pm 0.04$ & 105.86 & $1.29 \pm 0.06$ & 106.61 & $0.86 \pm 0.01$ & 104.88 \\
\hline & 10 & $2.77^{* *} \pm 0.36$ & 123.66 & $3.43^{*} \pm 0.08$ & 111.73 & $1.48^{*} \pm 0.03$ & 122.31 & $0.81 \pm 0.01$ & 98.78 \\
\hline & 50 & $3.26^{* *} \pm 0.05$ & 145.54 & $3.81^{* *} \pm 0.06$ & 124.10 & $1.52^{*} \pm 0.01$ & 125.62 & $0.75^{*} \pm 0.03$ & 91.46 \\
\hline & 100 & $3.53^{* * *} \pm 0.08$ & 157.59 & $4.58^{* * *} \pm 0.05$ & 149.19 & $1.57^{*} \pm 0.04$ & 129.75 & $0.77 \pm 0.03$ & 93.90 \\
\hline & 200 & $4.00^{* *} \pm 0.11$ & 178.57 & $6.15^{* * *} \pm 0.04$ & 200.33 & $2.10^{* * *} \pm 0.04$ & 173.33 & $0.81 \pm 0.01$ & 98.78 \\
\hline \multirow{6}{*}{$\begin{array}{l}\mathrm{Cu}^{+2}+200 \\
\mathrm{mg} \mathrm{L}^{-1} \\
\text { thiamine }\end{array}$} & 0 & $2.33 \pm 0.07$ & 104.02 & $3.01 \pm 0.09$ & 97.05 & $1.11 \pm 0.10$ & 91.74 & $0.77 \pm 0.01$ & 93.90 \\
\hline & 0.5 & $2.79^{* *} \pm 0.18$ & 124.55 & $3.41^{*} \pm 0.09$ & 111.07 & $1.39 \pm 0.03$ & 114.88 & $0.89^{*} \pm 0.01$ & 108.54 \\
\hline & 10 & $3.12^{* *} \pm 0.13$ & 139.29 & $3.72^{* *} \pm 0.09$ & 121.17 & $1.64^{* *} \pm 0.05$ & 135.54 & $0.96^{* * *} \pm 0.03$ & 117.07 \\
\hline & 50 & $3.54^{* *} \pm 0.11$ & 158.04 & $4.54^{* * *} \pm 0.27$ & 147.88 & $1.79^{* * *} \pm 0.03$ & 147.93 & $1.05^{* * *} \pm 0.06$ & 128.05 \\
\hline & 100 & $4.01^{* *} \pm 0.09$ & \begin{tabular}{|l|}
179.02 \\
\end{tabular} & $5.70^{* * *} \pm 0.13$ & 185.67 & $1.89^{* * *} \pm 0.03$ & 156.20 & $1.28^{* * *} \pm 0.02$ & 156.10 \\
\hline & 200 & $4.21^{* *} \pm 0.15$ & 187.45 & $6.63^{* * *} \pm 0.41$ & 215.96 & $2.07^{* * *} \pm 0.04$ & 171.07 & $1.45^{* * *} \pm 0.02$ & 176.83 \\
\hline \multirow{6}{*}{$\begin{array}{l}\mathrm{Cu}^{+2}+200 \\
\mathrm{mg} \mathrm{L}^{-1} \\
\text { pyridoxine }\end{array}$} & 0 & $2.27 \pm 0.06$ & 101.34 & $3.11 \pm 0.10$ & 101.30 & $1.19 \pm 0.07$ & 98.35 & $0.78 \pm 0.01$ & 95.12 \\
\hline & 0.5 & $3.05^{* * *} \pm 0.06$ & 136.16 & $3.73^{* * *} \pm 0.11$ & 121.50 & $1.58^{* * *} \pm 0.05$ & 130.58 & $0.96^{* * *} \pm 0.02$ & 117.07 \\
\hline & 10 & $3.31^{* *} \pm 0.20$ & 147.77 & $4.32^{* *} \pm 0.21$ & 140.72 & $1.79^{* *} \pm 0.09$ & 147.93 & $1.07^{* *} \pm 0.05$ & 130.49 \\
\hline & 50 & $3.70^{* *} \pm 0.19$ & 165.52 & $4.96^{* * *} \pm 0.29$ & 161.56 & $1.92^{* * *} \pm 0.12$ & 158.68 & $1.20^{* * *} \pm 0.02$ & 146.34 \\
\hline & 100 & $4.36^{* *} \pm 0.15$ & 194.64 & $6.03^{* *} \pm 0.21$ & 196.42 & $2.15^{* *} \pm 0.05$ & 177.69 & $1.44^{* *} \pm 0.07$ & 175.61 \\
\hline & 200 & $4.60^{* * *} \pm 0.09$ & 205.36 & $6.97^{* * *} \pm 0.06$ & 227.04 & $2.39^{* * *} \pm 0.02$ & 197.52 & $1.59^{* * *} \pm 0.02$ & 193.90 \\
\hline \multirow[t]{2}{*}{ L.S.D } & $5 \%$ & 0.24 & & 0.28 & & 0.27 & & 0.06 & \\
\hline & $1 \%$ & 0.33 & & 0.37 & & 0.36 & & 0.08 & \\
\hline
\end{tabular}

*Significant differences at $(\mathrm{p}=0.05)$ level $* *$ highly significant differences from control at $(\mathrm{p}=0.01)$. $\pm \mathrm{SD}, \mathrm{n}=3$ 
A pronounced additional stimulation in the activities of SOD, CAT, POD and APX was observed as a result of vitamin treatments; especially at high levels of $\mathrm{Cu}^{+2}$. The stimulation effect was more pronounced in pyridoxine than in thiamine treated algae (Table 4).

\section{Discussion}

The present study indicated that application of low $\mathrm{Cu}^{+2}$ concentration $(0.5$ $\mu \mathrm{M})$ to $C$. vulgaris led to slight increases in growth criteria (growth rate \& dry weight) and pigment contents. On the other hand, progressive increases in $\mathrm{Cu}^{+2}$ concentration caused gradual reduction in these values. Such biphase response to copper was also revealed by other investigators (Deef, 2007 and Gao et al., 2008).

The reduction in growth could be due to inhibition of normal cell division by the metal, as has been reported for Spirulina platensis-S5.exposed to copper (Choudhary et al., 2007). The decrease in the rate of cell division caused by metals is primarily attributed to their binding to sulfhydryl groups which are important for regulating the plant cell division (Visviki \& Rachlin, 1991).

Three reasons may be responsible for the inhibitory effect on Chl. a, Chl. b and carotenoids seen in excess $\mathrm{Cu}^{2+}$. First, $\mathrm{Cu}^{2+}$ probably induces production of reactive oxygen species and inhibits the reductive steps in the biosynthesis pathway of these pigments (Clijsters et al., 1999). Second, $\mathrm{Cu}^{2+}$ can directly destroy the structure and function of chloroplast by binding with $\mathrm{SH}$ group of enzymes and overall chlorophyll biosynthesis (Singh, 1995). Third, it may activate pigment enzyme and accelerate the decomposition of pigment (Hou et al., 2007). Moreover, carotenoids appeared to be more resistant to $\mathrm{Cu}^{2+}$ phytotoxicity than Chl.a and Chl. b (Li et al., 2010).

Supplementary thiamine or pyridoxine resulted in a considerable increased in growth criteria (rate \& dry weight) and pigment contents of the tested alga and thus partially alleviated the toxic effects of $\mathrm{Cu}^{+2}$ as compared to the reference controls (Hamada, 2001 and Desouky et al., 2011).

In the present study the effect of different concentrations of $\mathrm{Cu}^{2+}$ on photosynthetic $\mathrm{O}_{2}$ evolution showed a tendency towards reducing the amount of $\mathrm{O}_{2}$ evolved by test alga in response to $\mathrm{Cu}^{2+}$. However, an increase in $\mathrm{O}_{2}$ evolution by $C$. vulgaris was observed at lower $\mathrm{Cu}^{2+}$ concentrations. The magnitude of the inhibitory action was found to increase with higher metal concentrations.

It is clear that the photosynthetic process depends on the content of pigments, which had been inhibited at the higher concentrations of $\mathrm{Cu}^{2+}$. Moreover, increased generation of reactive oxygen species induced by this metal can induce membrane lipid peroxidation and increase unstacking of thylakoids in Scenedesmus incrassatulus (Perales-vela et al., 2007).

Egypt. J. Microbiol. 47 (2012) 
Respiration (dark $\mathrm{O}_{2}$-uptake) of C. vulgaris cultures gradual decreased at lower $\mathrm{Cu}^{+2}$ levels, then a highly significant increased by increasing the $\mathrm{Cu}^{2+}$ in alga cultures (Andrade et al., 2004).

Application of both vitamins (either thiamine or pyridoxine) induces a marked stimulation in $\mathrm{O}_{2}$-evolution compared to the reference controls. In contrast, $\mathrm{O}_{2}$-uptake was retarded at the lower and moderate $\mathrm{Cu}^{+2}$ levels, and then activated at the higher levels compared to the absolute control (Desouky, 2011).

The result suggested that accumulation of proline responds to $\mathrm{Cu}^{+2}$ and it varies with respect to the toxicity caused by $\mathrm{Cu}^{+2}$ treatment. Proline accumulation prevents membrane distortion and acts as a hydroxyl radical scavenger. Mehta \& Gaur (1999) also note a protective role of proline in mitigating metal-induced lipid peroxidation in C. vulgaris. Thus greater accumulation of proline under high $\mathrm{Cu}^{+2}$ level of the present study suggested the protective role of proline to the alga to survive $\mathrm{Cu}^{+2}$ stress (Choudhary et al., 2007).

Our results indicate that concentrations of $\mathrm{Cu}^{2+}$ increases oxidative damage as is evident from increased lipid peroxidation. Thus, the increased level of MDA suggests that metal ion stimulate free radical generating capacity of the microorganism. It is accordance with the previous findings (Thounaojam et al., 2012) that MDA accumulated greatly after the exposure of $\mathrm{Cu}^{+2}$ and cell membrane is the primary site of $\mathrm{Cu}^{+2}$ toxicity. It might be due to the overproduction of ROS under $\mathrm{Cu}^{+2}$ stress, which is highly destructive to cell membrane.

Increase in both proline and MDA contents with increasing $\mathrm{Cu}^{+2}$ concentrations are indicative of a correlation between free radical generation and proline accumulation. Our study also depicted an inverse relationship between growth criteria and proline accumulation in the test alga under $\mathrm{Cu}^{+2}$ oxidative stress (Fig.1a, b). This might involve reduction in cell division or delay of exponential growth due to proline accumulation (Maggio et al., 2002).
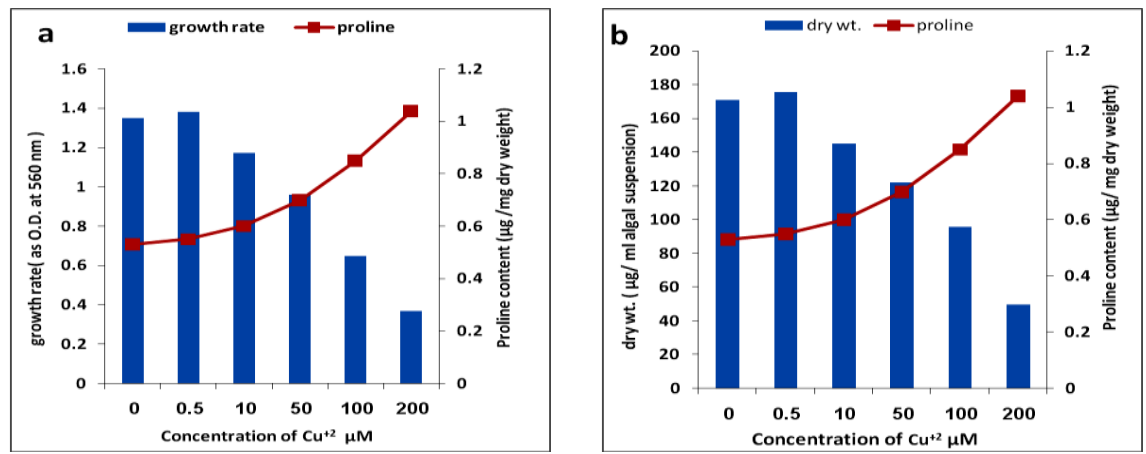

Fig.1. Correlation between proline accumulation and growth criteria (growth rate a and dry weight b) of $C$.vulgaris under $\mathrm{Cu}^{+2}$ stress.

Antioxidant enzymes play important roles in defense mechanisms may provide a strategy to enhance oxidative stress tolerance. In the present study $\mathrm{Cu}^{+2}$

Egypt. J. Microbiol. 47 (2012) 
treatment resulted in a marked and progressive increase in the activities of SOD, CAT \& POD, which can be considered as indicators for evidence of enhanced production of reactive oxygen species, such as the superoxide radical $\left(\mathrm{O}_{2}\right)$, hydrogen peroxide $\left(\mathrm{H}_{2} \mathrm{O}_{2}\right)$ and hydroxyl radical $\left(\mathrm{HO}^{-}\right)$under $\mathrm{Cu}^{+2}$ stress (Verma et al., 2011 and Thounaojam et al., 2012). On the other hand, the APX activity remained around the absolute control value at the all levels of $\mathrm{Cu}^{+2}$.

Stimulation of antioxidant enzymes reflects the ability of the $C$. vulgaris to withstand the $\mathrm{Cu}^{+2}$ induced oxidative stress. Proline accumulation also appears to be an additional defense against $\mathrm{Cu}^{+2}$ oxidative stress.

Thiamine or pyridoxine treatments induced pronounced inhibition in the accumulation of proline and MDA content when compared to algae treated with only $\mathrm{Cu}^{+2}$ (Al-Hakimi \& Hamada, 2011). This confirmed the alleviating capacity of these vitamins on the algal growth. In contrast, antioxidant enzymes activities increased markedly, thus improving alga resistance.

\section{Conclusion}

The growth of $C$. vulgaris appears biphase response to copper and exogenous thiamine or pyridoxine partially alleviated the toxic effects of $\mathrm{Cu}^{+2}$ in the growth criteria by promoting photosynthetic rate and antioxidant enzymes activities (SOD, CTA, POD \& APX) which are associated with a marked retardation in proline and MDA contents, and consequently stimulate the alga growth.

\section{References}

Aebi, H. (1984) Catalase in vitro. Meth. Enzymol. 105, 121-126.

Al-Hakimi, A.M. and Hamada A.M. (2011) Ascorbic acid, thiamine or salicylic acid induced changes in some physiological parameters in wheat grown under copper stress. Plant Protect. Sci. 47 (3), 92-108.

Alia, S.P.P., Mohanty, P. and Matysik, J. (2001) Effect of proline on the production of singlet oxygen. Amino Acid, 21, 195-200

Andrade, L. R., Farina, M. and Filho A. M. G. (2004) Effects of copper on Enteromorpha flexuosa (Chlorophyta) in vitro. Ecotoxicol. Environ. Safe. 58, 117125.

Asada, K. and Chen, G. (1992) Inactivation of ascorbate peroxidase by thiols requires hydrogen peroxide. Plant Cell Physiol. 33, 117-123.

Bates, L.S., Waldren, R.P. and Tear, L.D. (1973) Rapid determination of free proline for water-stress studies. Plant Soil, 39, 205-207.

Bischoff, H.W. and Bold, H.C. (1963) Phycological studies. for some soil algae from enchanted rock and related algal species. Univ., Texas. Publ. 6318, pp. 32-36.

Egypt. J. Microbiol. 47 (2012) 
Brownleader, M.D., Hopkins, J., Mobasheri, A., Dey, P.M., Jackson P. and Trevan, M., (2000) Role of extension peroxidase in tomato (Lycopersicon esculentum Mill.) seedling growth. Planta, 210, 668-676.

Chang, C. and Sibley, T. H. (1993) Accumulation and transfer of copper by Oocystis pusilla. Bull Environ. Contamin. Toxicol., 50, 689- 695.

Choudhary, M., Jetley, U.K., Khan, M.A., Zutshi, S. and Fatma, T. (2007) Effect of heavy metal stress on proline, malondialdehyde, and superoxide dismutase activity in the cyanobacterium Spirulina platensis-S5. Ecotoxicol. Environ. Safety, 66, 204-209.

Clijsters, H., Cuypers, A. and Vangronsveld, J. (1999) Physiological response to heavy metals in higher plants defense against oxidative stress. Z. Naturf. 54, 730-734.

Deef, H.E. (2007) Cupper treatments and their effects on growth, carbohydrates, mineral and essential oils contents of Rosmarinus officinalis L. World Journal of Agricultural Sciences, 3 (3), 322-328.

Desouky, S.A. (2011) Effect of some natural organic additives on the growth and photosynthesis of pollutant - Chlorella vulgaris Beijer. J. Applied Sciences Research, 7(1), 23-32.

Desouky, S.A., Usama, M. A. and Ahmed, W. A. (2011) Effect of vitamins on growth criteria, photosynthetic pigments and some metabolic products of cobalt chloride stressed Scenedesmus obliquus cultures. Ass. Univ. Bull. Environ. Res. 14 (2).

Dhindsa, R.S. and Matowe, W. (1981) Drought tolerance in two mosses: correlated with enzymatic defense against lipid peroxidation. J. Exp. Bot. 32, 79-91.

Fang, W.C. and Kao, H.C. (2000) Enhanced peroxidase activity in rice leaves in response to excess iron, copper, and zinc. Plant. Sci. 158, 71-76.

Gao, S., Yan, R.,Cao, M., Yang, W., Wang,S. and Chen, F. (2008) Effects of copper on growth, antioxidant enzymes and phenylalanine ammonia-lyase activities in Jatropha curcus L. seedling. Plant Soil Environ. 54(3), 117-122.

Gupta, U.C. and Kalra, Y.P. (2006) Residual effect of copper and zinc from fertilizers on plant concentration, phytotoxicity and crop yield response. Commun. Soil Sci. Plant Anal., 37, 2505-2511.

Hamada, A.M. (2001) Vitamin or salicylic acid treatments versus the adverse effects of copper stress on photosynthesis and some related activities of wheat seedlings. In Proceeding: XIIth International Photosynthesis Congress. Brisbane, Australia, pp. 18-23.

Hassanein, R.A., Bassuony, F.M., Baraka, D.M. and Khalil, R.R. (2009) Physiological effects of nicotinamide and ascrobic acid on Zea mays plant grown under salinity stress. I- Changes in growth, some relevant metabolic activities and oxidative defense systems. Res. J. Agri. \& Biol.Sci. 5(1), 72-81.

Havir, E.A. and Mellate, N.A. (1987) Biochemical and developmental characterization of multiple forms of catalase in tobacco leaves. Plant Physiol. 84, 450-455. 
Heath, R.L. and Packer, L. (1968) Photoperoxidation in isolated chloroplast. I. Kinetics and stiochiometry of fatty acid peroxidation. Arch. Bioch. Biophys. 125, 189-198.

Hou, W. H., Song, G. L., Wang, Q. H. and Chang, C. C. (2007) Effects of copper and cadmium on heavy metal polluted water body restoration by duckweed (Lemna minor). Pl. Physiol. Biochem. 45, 2-69.

Joseph, B. and Jini, D. (2010) Insight into the role of antioxidant enzymes for salt tolerance in plants. Int. J. Bot. 6, 456-464.

Kadpal, R.P. and Rao, N.A. (1985) Alteration in the biosynthesis of proteins and nucleic acid in finger millet (Eleucine coracana) seedling during water stress and the effect of proline on protein biosynthesis. Plant Sci. 40, 73-79.

Kawasaki, T. (1992) "Modern Chromatographic Analysis of Vitamins". $2^{\text {nd }}$ ed., New York, NY: Marcel Dekker, Inc., 60, pp. 319-354.

Küpper, H., Šetlík, I., Spiller, M., Küpper, F. C. and Prášil, O. (2002) Heavy metalinduced inhibition of photosynthesis: targets of in vivo heavy metal chlorophyll formation. J. Phycol. 38, 429- 441.

Laliberte, G. and Hellebust, J.A. (1989) Regulation of proline content of Chlorella autopica in response to change in salinity. Can. J. Bot. 67, 1959-1965.

Levy, J.L., Stauber, J.L. and Jolley, D.F. (2007) Sensitivity of marine microalgae to copper: the effect of biotic factors on copper adsorption and toxicity. Sci. Total Environ. 387, 141-154.

Li, Y. X., Zhou, S., Zhao, F. J., Liu, Y., Fan, P. P. and Wang G. C. (2010) Physiological responses of Porphyra haitanesis to different copper and zinc concentrations. Brazilian Journal of Oceanography, 58(4), 261-267.

Maehly, A.C. and Chance, B. (1954) The assay of catalase and peroxidase. Meth. Anal. Bioch. (D. Glick,.). 1, 357.

Maggio, A., Miyazaki, S., Veronese, P., Fujita, T., Ibeas, J.I., Damsz, B., Narasimhan, M.L., Hasegawa, P.M., Joly, R.J. and Bressan, R.A. (2002) Dose proline accumulation plays an active role in stress-induced growth reduction. Plant J. 31 (6), 699-712.

Mehta, S.K. and Gaur, J.P. (1999) Heavy metal-induced proline accumulation and its role in ameliorating metal toxicity in Chlorella vulgaris, New Phytol. 143, 253-259.

Metzner, H., Rau, H. and Senger, H. (1965) Untersuchungen zur Synchronisier barkeit einzelner pigment mangel mutanten von Chlorella. Planta, 65, 186-194

Mukherjee, S.P. and Choudhuri, M.A. (1983) Implications of water stress-induced changes in the levels of endogenous ascorbic acid and hydrogen peroxide in Vigna seedlings. Physiologia Plantarum, 58, 166-170.

Nikolopoulos, D. and Manetas, Y. (1991) Compatible solutes and in vitro stability of Salsola soda enzyme: proline incompability. Phytochemistry, 30, 411-413.

Egypt. J. Microbiol. 47 (2012) 
Ohkawa, H., Ohishi, N. and Yagi, K. (1979) Assay for lipid peroxidation in animal tissues by thiobarbituric acid reaction. Anal. Biochem. 95, 351 .

Passsardi, F., Penel, C. and Dunand, C. (2004) Performing the paradoxical: How plant peroxidase modify the cell wall. Trends Plant Sci. 9, 534-540.

Perales-vela, H.V., González-moreno, S., Montes-horcasitas, C. and Caňizaresvillanueva, R. O. (2007) Growth, photosynthetic and respiratory responses to sub-lethal copper concentrations in Scenedesmus incrassatulus (Chlorophyceae). Chemosphere, 67, 22742281.

Singh, V.P. (1995) Toxic metal cadmium: phytotoxicity and tolerance in plants. In: "Advances in Environmental Science Technology". Trivedy, R. K. (Ed.). pp. 225-256. New Delhi: Ashish Publication House.

Smirnoff, N. and Cumbes, Q.J. (1989) Hydroxyl radical scavenging activity of compatible solute. Phytochemistry, 28,1057-1060.

Thounaojam, T.C., Pandaa, P., Mazumdar, P., Kumar, D., Sharma, G.D., Sahoo, L. and Panda, S.K. (2012) Excess copper induced oxidative stress and response of antioxidants in rice. J. Plant Physiology \& Biochemistry, 53, 33-39.

Venekemp, J.H., (1989) Regulation of cytosolic acidity in plants under condition of drought. Plant Physiol. 76, 112-117.

Verma, J. P., Singh, V. and Yadav, J. (2011) Effect of copper sulphate on seed germination, plant growth and peroxidase activity of Mung Bean (Vigna radiate). Intr. J. Bot. 7(2), 200-204.

Visviki, I. and Rachlin, J.W. (1991) The toxic action and interactions of copper and cadmium to the marine alga Dunaliella minuta in both acute the chronic exposure. Arch. Environ. Contam. Toxicol. 2, 271-275.

Wang, S.H., Yang, Z.M., Lu, B., Li, S.Q. and Lu, Y.P. (2004) Copper induced stress and antioxidative responses in roots of Brassica juncea L. Bot. Bull. Acad. Sin. 45, 203-212.

Wetherel, D.F. (1961) Culture of fresh water algae in enriched natural sea water. Physiol Plant. 14, 1-6. 


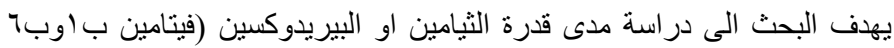

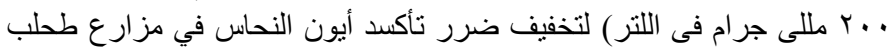

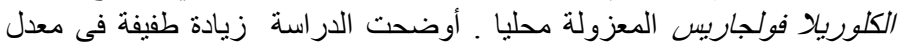

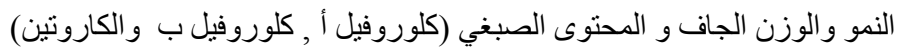

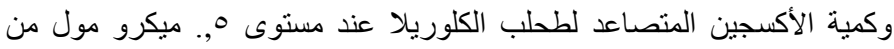

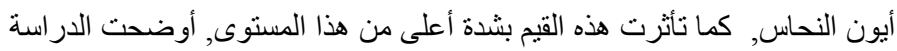

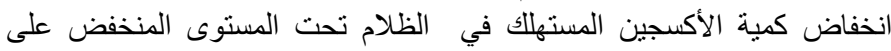

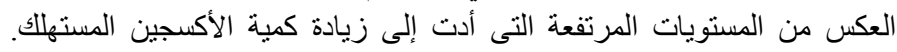

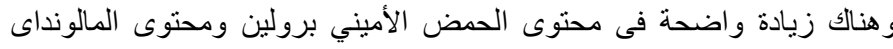

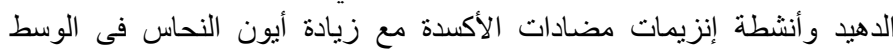

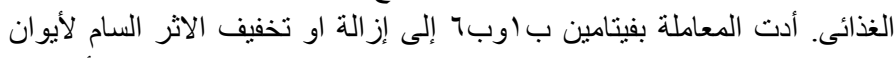

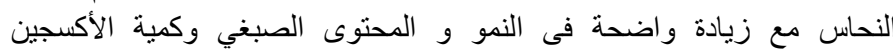

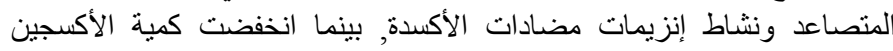

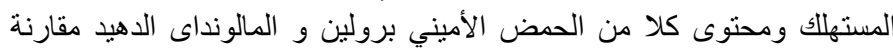
بمثيلاتها المعاملة فقط بالنحاس. 